\title{
The Spin Structure of the Nucleon
}

\author{
G.K. Mallot \\ EP Division, CERN \\ CH-1211 Geneva, Switzerland
}

\section{Introduction}

The discovery [1, 2] by the European Muon Collaboration (EMC) that the axial charge of the proton is much smaller than expected from the Ellis-Jaffe sum rule [3] implies that the nucleon spin is not simply made up by the quark spins. This surprising result was confirmed by a series of experiments: the Spin Muon Collaboration experiment at CERN [4, 5, 6], the SLAC experiments E142 [7], E143 [8], E154 [9, 10], E155 [11, 12] and the Hermes experiment [13, 14, 15] at DESY. They increased the kinematic range and the precision of the data to a level where QCD analyses begin to become a powerful tool like in the unpolarised case.

Albeit the immense theoretical progress and the wealth of data the original problem remains that the origin of the nucleon's spin is not yet understood. Apart from the quark spins, $\Delta \Sigma=\Delta u+\Delta d+\Delta s$, the gluon spin, $\Delta g$, and orbital angular momentum, $L$, must play a major rôle in making up the nucleon spin

$$
\frac{1}{2}=\frac{1}{2} \Delta \Sigma+L_{q}+\Delta g+L_{g}
$$

It is well known from both, experiment and theory, that at high $Q^{2}$ about half of the nucleon's longitudinal momentum is carried by the gluons. The same sharing was predicted for the total angular momentum of the nucleon [16]. In the Quark Parton Model the polarised quark distribution functions,

$$
\Delta q=\left(q^{+}-q^{-}\right)+\left(\bar{q}^{+}-\bar{q}^{-}\right),
$$

are related to the spin-dependent structure function $g_{1}$ by

$$
g_{1}\left(x, Q^{2}\right)=\frac{1}{2} \sum_{f} e_{f}^{2} \Delta q_{f}\left(x, Q^{2}\right),
$$

where $f$ runs over the quark flavours and $e_{f}$ are the electrical quark charges. The notations $q^{+(-)}$refer to parallel (antiparallel) orientation of the quark and nucleon spins.

Invited talk presented on the XIX International Symposium on Lepton and Photon Interactions, August 9-14, 1999, Stanford University, CA 
Experimentally the spin-dependent structure functions, $g_{1}$ and $g_{2}$, are obtained from the measured event-number asymmetries, $A_{\|}^{\text {raw }}$, for longitudinal orientation of the target and lepton spins, and $A_{\perp}^{\text {raw }}$ for transverse target polarisations. These raw asymmetries range for the proton typically from a few per cent at large $x$ to a few parts per thousand at small $x$. In the lepton-nucleon asymmetries, $A_{\|}$and $A_{\perp}$, the uncertainties of the raw asymmetries get amplified by the factor $1 / P_{b} P_{t} f$ accounting for the incomplete beam and target polarisations, $P_{b}$ and $P_{t}$, and the dilution factor, $f$. Typical target materials contain a large fraction of unpolarisable nucleons and $f$ denotes the fraction of the total spin-averaged cross section arising from the polarisable nucleons. For the target materials used, $f$ varies from about 0.13 (butanol), over 0.17 (ammonia) to $0.3\left({ }^{3} \mathrm{He}\right)$. For deuterated butanol and ammonia $f$ is 0.23 and 0.3 , respectively, while for the proton and deuteron gas targets $f$ is close to unity. The neutron structure functions are either obtained from the combination of proton and deuteron data or from experiments using ${ }^{3} \mathrm{He}$ targets. The deuteron asymmetries are slightly reduced from the average of proton and neutron asymmetries due to the D-state component in the deuteron wave function. The ${ }^{3} \mathrm{He}$ asymmetry is mainly due to the unpaired neutron, however a small proton contribution has to be corrected for. Due to the cancellation of the isotriplet part in $g_{1}^{\mathrm{d}}$ the measurements using deuteron targets are most sensitive to the flavour-singlet part and thus to the Ellis-Jaffe sum rule. The structure functions, $g_{1}$ and $g_{2}$, are related to the virtual photon asymmetries, $A_{1}$ and $A_{2}$, via

$$
\begin{array}{lll}
A_{\|}=D\left(A_{1}+\eta A_{2}\right), & A_{\perp}=d\left(A_{2}-\xi A_{1}\right), \\
A_{1}=\frac{g_{1}-\gamma^{2} g_{2}}{F_{1}}, & A_{2}=\gamma \frac{g_{1}+g_{2}}{F_{1}} .
\end{array}
$$

Here $F_{1}=F_{2}\left(1+\gamma^{2}\right) / 2 x(1+R)$ is the well-known spin-averaged structure function and $\gamma^{2}=Q^{2} / \nu^{2}, \eta$, and $\xi$ are kinematic factors, which are small in most of the kinematic domain covered by the data. The variables $\nu$ and $Q^{2}=-q^{2}$ denote respectively the energy transfer and negative square of the 4-momentum transfer. The kinematic factors, $D$ and $d$, account for the incomplete transverse polarisation of the virtual photon. With longitudinal target polarisation predominantly $g_{1}$ is determined, while experiments with transverse target polarisation are sensitive to $g_{1}+g_{2}$. The virtual photon asymmetries are bounded by $\left|A_{1}\right| \leq 1$ and $\left|A_{2}\right| \leq \sqrt{R}$, where $R=\sigma_{L} / \sigma_{T}$ is the longitudinal-to-transverse photoabsorption cross-section ratio known, like $F_{2}$, from unpolarised deep inelastic scattering. 


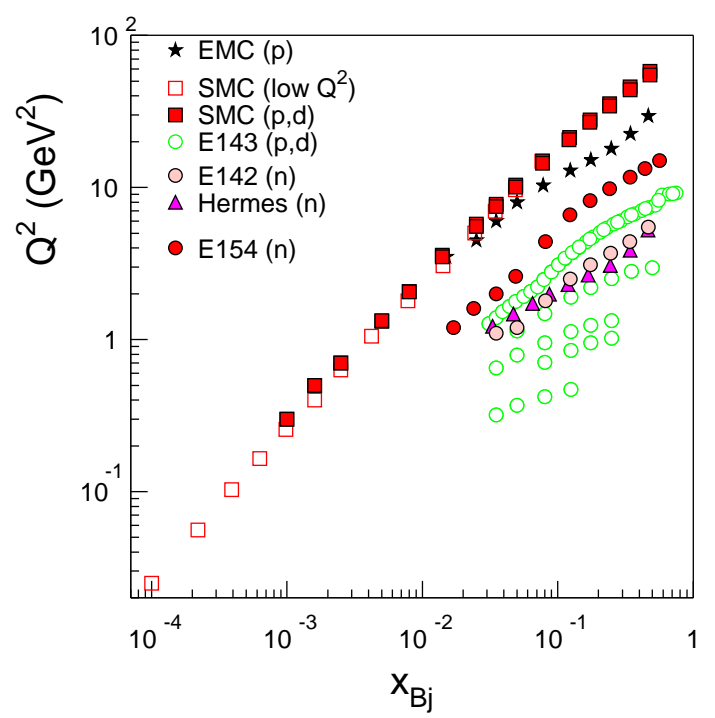

Figure 1: Kinematic ranges of the individual experiments. The double logarithmic scale emphasises the new data in the small- $x$, small- $Q^{2}$ region from a special SMC trigger.

\section{Experiments and status of structure function data}

The experimental approach of the three recent series of experiments on the structure function $g_{1}$ is rather different. The highest momentum transfers, $Q^{2}$, and the lowest values of $x$-Bjorken were reached by the SMC experiments at the $190 \mathrm{GeV}$ CERN muon beam. The rather low intensity of muon beams required thick solid-state polarised targets. The two target cells allow a simultaneous measurement of targets with opposite polarisations [17]. Both, butanol $(\mathrm{p}, \mathrm{d})$ and ammonia $(\mathrm{p})$ targets were used. The to date most precise data come from the SLAC experiments E154 and E155, which followed the earlier E142 and E143 experiments. They were performed at the high-intensity, $49 \mathrm{GeV}$ SLAC electron beam with a thin ${ }^{3} \mathrm{He}$ gas target (E154) and solid state $\mathrm{NH}_{3}$ and LiD targets (E155) 18. Due to the possible rapid change in beam polarisation only one target is needed. Several magnetic spectrometers determined the momentum of the scattered electrons. The advantage of the internal gas target of Hermes [19] in the Hera electron storage ring is its low mass which enables this experiment to obtain precise semi-inclusive data, where in addition to the scattered lepton additional hadrons are detected. Another advantage is the absence of 
Table 1: Parameters of the experiments

\begin{tabular}{llrrrcc}
\hline \hline Experiment & Lab. & Beam & $\begin{array}{c}\left\langle Q^{2}\right\rangle \\
{\left[\mathrm{GeV}^{2}\right]}\end{array}$ & $x$ range & Targets \\
\hline E-80/130 & SLAC & $22 \mathrm{GeV}$ & $\mathrm{e}$ & 4 & $0.18 \leq x \leq 0.7$ & $\mathrm{p}$ \\
EMC & CERN & $200 \mathrm{GeV}$ & $\mu$ & 10 & $0.01 \leq x \leq 0.7$ & $\mathrm{p}$ \\
SMC & CERN & $190 \mathrm{GeV}$ & $\mu$ & 10 & $0.003 \leq x \leq 0.7$ & $\mathrm{p}, \mathrm{d}$ \\
E-142 & SLAC & $29 \mathrm{GeV}$ & $\mathrm{e}$ & 3 & $0.03 \leq x \leq 0.8$ & ${ }^{3} \mathrm{He}$ \\
E-143 & SLAC & $29 \mathrm{GeV}$ & $\mathrm{e}$ & 3 & $0.03 \leq x \leq 0.8$ & $\mathrm{p}, \mathrm{d}$ \\
E-154 & SLAC & $48 \mathrm{GeV}$ & $\mathrm{e}$ & 5 & $0.014 \leq x \leq 0.7$ & ${ }^{3} \mathrm{He}$ \\
E-155 & SLAC & $48 \mathrm{GeV}$ & $\mathrm{e}$ & 5 & $0.01 \leq x \leq 0.9$ & $\mathrm{p}, \mathrm{d}\left({ }^{6} \mathrm{LiD}\right)$ \\
Hermes & DESY & $27 \mathrm{GeV}$ & $\mathrm{e}$ & 3 & $0.023 \leq x \leq 0.6$ & ${ }^{3} \mathrm{He}, \mathrm{p}, \mathrm{d}$ \\
\hline \hline
\end{tabular}

unpolarised nucleons in the hydrogen and deuterium gas targets. Two spin rotators before and after the detector provide parallel or antiparallel longitudinal polarisation of the electrons at the interaction point. The polarisation of the gas target can be inverted within milliseconds. The rather low energy of the Hera electron beam of $27 \mathrm{GeV}$ makes the interpretation of the data not always straight forward. The kinematic coverage of these experiments shown in Fig. 1 reflects the different energies of the incident leptons.

The asymmetries $g_{1} / F_{1}\left(x, Q^{2}\right)$ as compiled by the E155 collaboration are shown for $Q^{2}>1 \mathrm{GeV}^{2}$ in Fig. 2 [11, 20]. For the proton an indication of a positive $Q^{2}$ dependence is visible in the small- $Q^{2}$ region, while the deuteron asymmetries show no such trend. In order to compare the data taken at different momentum transfers, the structure function data, $g_{1}\left(x, Q^{2}\right)$, were evolved to a common value of $Q^{2}=5 \mathrm{GeV}^{2}$. The results for the proton, the deuteron and the neutron are shown in Figs. 3. All data sets are in good agreement. While for the proton there is (not yet) an indication of a decreasing $g_{1}$ towards small $x$ as expected from QCD evolution, this effect is clearly visible for the neutron.

Recently the SMC has published data down to $x=6 \cdot 10^{-5}$, where the corresponding momentum transfer is only $Q^{2}=0.01 \mathrm{GeV}^{2}$ [5]. These data were taken with a special low- $x$ trigger. The large radiative corrections at low $x$ were suppressed by requiring the presence of an additional hadron. This requirement also reduces the contamination by events from muon-electron scattering, which appear at $x=m_{\mathrm{e}} / m_{\mu}$. Additional kinematic cuts were applied to further reduce this background. The asymmetries and structure functions taken with this special low- $x$ trigger are shown together with those from the standard physics triggers in Figs. 因 and 5. The structure functions in the new low- $x$ region do not exhibit any unexpected behaviour. 

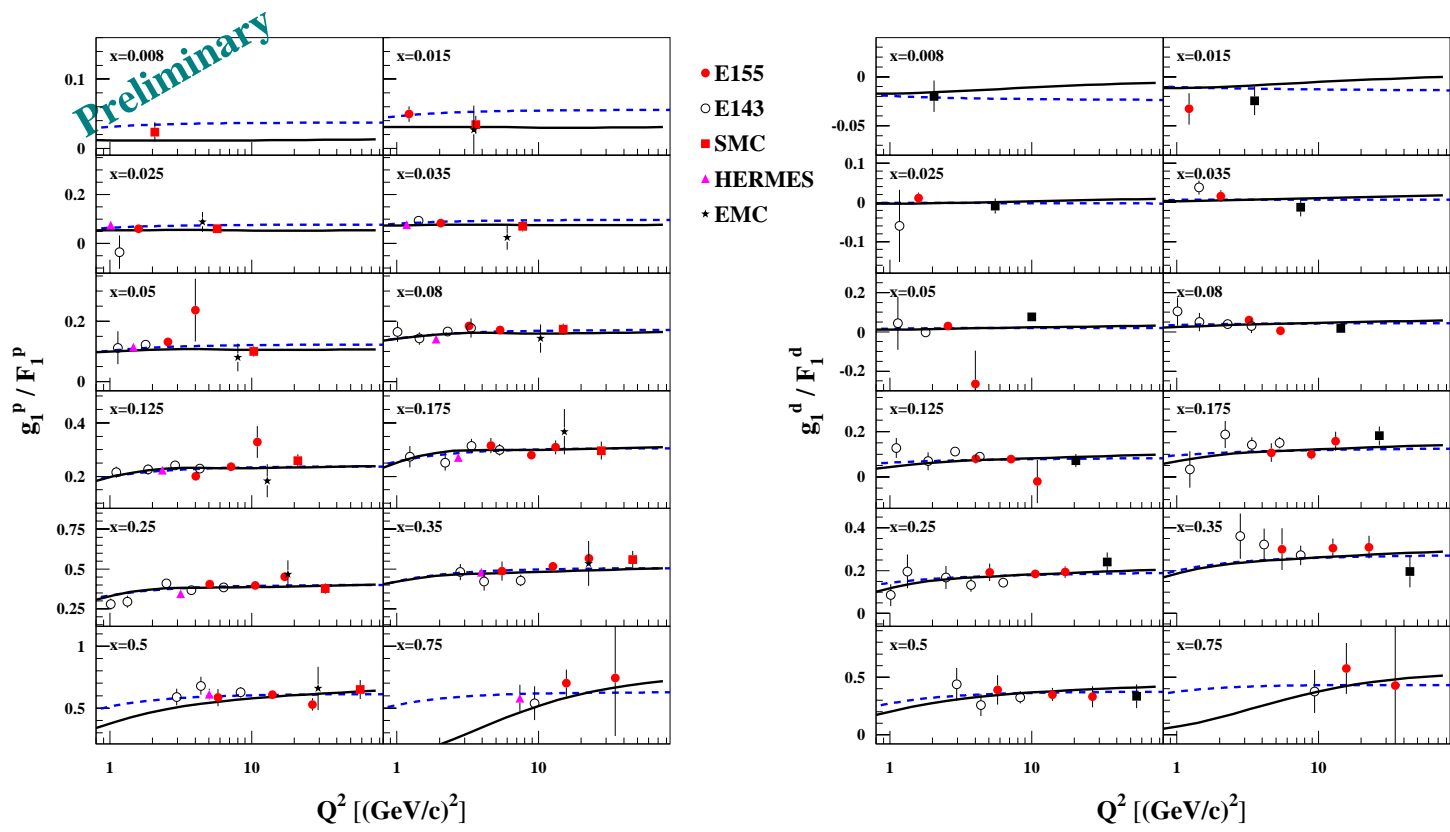

Figure 2: Proton (left) and deuteron structure functions $g_{1}\left(x, Q^{2}\right)$ for $Q^{2}>1 \mathrm{GeV}^{2}$. The proton data from E155 are preliminary. Also shown are a phenomenological fit to the data (dashed) and the E154 QCD-fit (solid) 21]

\section{QCD analyses of $g_{1}$ data}

As in the unpolarised case the $Q^{2}$ evolution of the structure functions is predicted by QCD. With the increasing precision of the data and after the next-to-leading order splitting functions were calculated [22, 23] QCD analyses have become a powerful tool in the understanding of polarised structure function and parton distribution functions.

The evolution equations are expressed in terms of the polarised splitting functions, $\Delta P$, and the singlet and non-singlet quark distribution functions, $\Delta \Sigma$ and $\Delta q^{\text {ns }}$

$$
\begin{aligned}
\frac{\mathrm{d}}{\mathrm{d} t} \Delta q^{\mathrm{ns}} & =\frac{\alpha_{s}(t)}{2 \pi} \Delta P_{q q}^{\mathrm{ns}} \otimes \Delta q^{\mathrm{ns}} \\
\frac{\mathrm{d}}{\mathrm{d} t}\left(\begin{array}{c}
\Delta \Sigma \\
\Delta g
\end{array}\right) & =\frac{\alpha_{s}(t)}{2 \pi}\left(\begin{array}{cc}
\Delta P_{q q}^{\mathrm{s}} & 2 n_{f} \Delta P_{q g}^{\mathrm{s}} \\
\Delta P_{g q}^{\mathrm{s}} & \Delta P_{g g}^{\mathrm{s}}
\end{array}\right) \otimes\left(\begin{array}{c}
\Delta \Sigma \\
\Delta g
\end{array}\right)
\end{aligned}
$$

with $t=\ln Q^{2} / \Lambda^{2}$ and the number of active flavours, $n_{f}$. The evolution of $\Delta \Sigma$ mixes with that of the polarised gluon distribution function, $\Delta g$. The structure function $g_{1}$ is then given by 

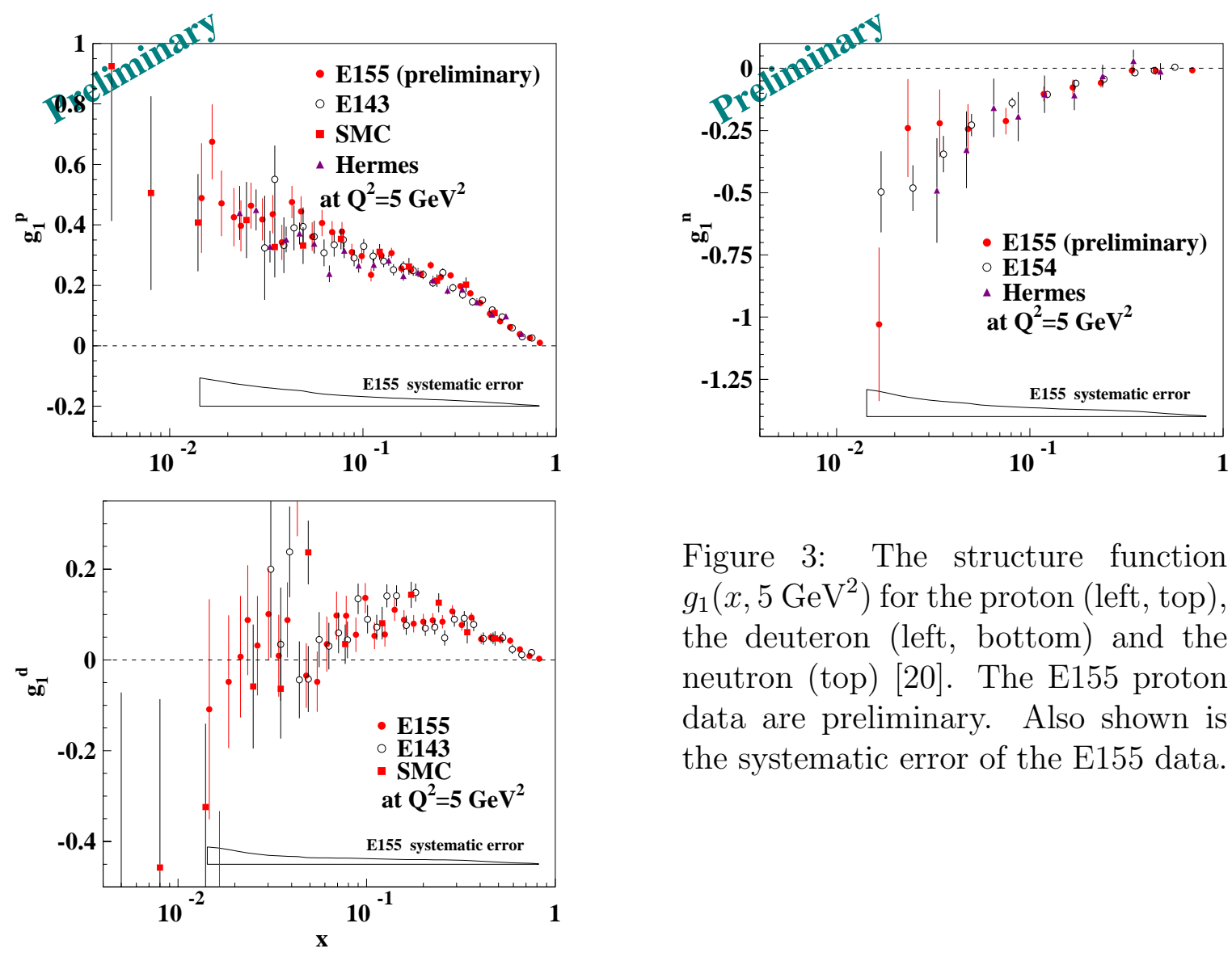

Figure 3: The structure function $g_{1}\left(x, 5 \mathrm{GeV}^{2}\right)$ for the proton (left, top), the deuteron (left, bottom) and the neutron (top) [20]. The E155 proton data are preliminary. Also shown is the systematic error of the E155 data.

$$
g_{1}^{\mathrm{p}, \mathrm{n}}=\frac{1}{12}\left( \pm \Delta q_{3}+\frac{1}{3} \Delta q_{8}\right) \otimes C^{\mathrm{ns}}+\frac{1}{9}\left(\Delta \Sigma \otimes C^{\mathrm{s}}+\Delta g \otimes 2 n_{f} C^{\mathrm{g}}\right)
$$

with the Wilson coefficients, $C$. The plus and minus symbols refer to the proton and the neutron, respectively. The non-singlet parton distribution functions are given by

$$
\begin{aligned}
& \Delta q_{3}\left(x, Q^{2}\right)=\Delta u\left(x, Q^{2}\right)-\Delta d\left(x, Q^{2}\right), \\
& \Delta q_{8}\left(x, Q^{2}\right)=\Delta u\left(x, Q^{2}\right)+\Delta d\left(x, Q^{2}\right)-2 \Delta s\left(x, Q^{2}\right) .
\end{aligned}
$$

The QCD fit is then performed in a particular renormalisation and factorisation scheme using parametrisations for $\Delta \Sigma, \Delta q^{\text {ns }}$ and $\Delta g$ at an initial value of $Q^{2}=Q_{i}^{2}$. The fit can either be performed in $x$ or in moment space ( $n$ space).

The difference of the two frequently used schemes, the $\overline{\mathrm{MS}}$ and the Adler-Bardeen (AB) scheme, is best illustrated on the first moment of $g_{1}$

$$
\Gamma_{1}\left(Q^{2}\right)=\int_{0}^{\infty} g_{1}\left(x, Q^{2}\right) \mathrm{d} x .
$$



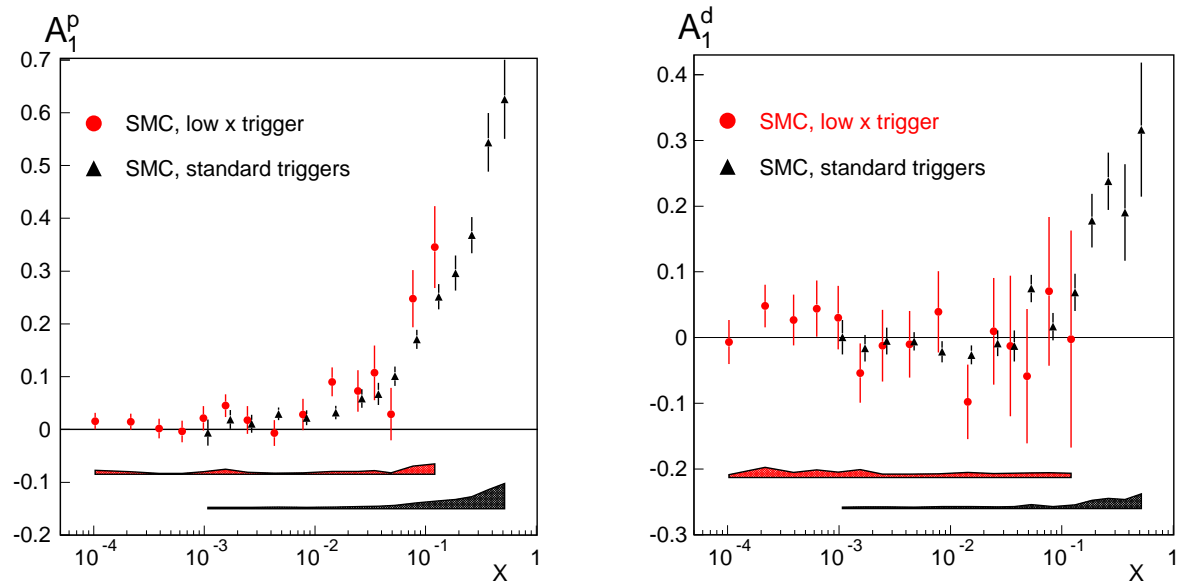

Figure 4: Asymmetries, $A_{1}(x)$, for the proton (left) and the deuteron (right) from the SMC low- $x$ and standard triggers [5]. The small- $x$ trigger extends to $x=6 \cdot 10^{-5}$.
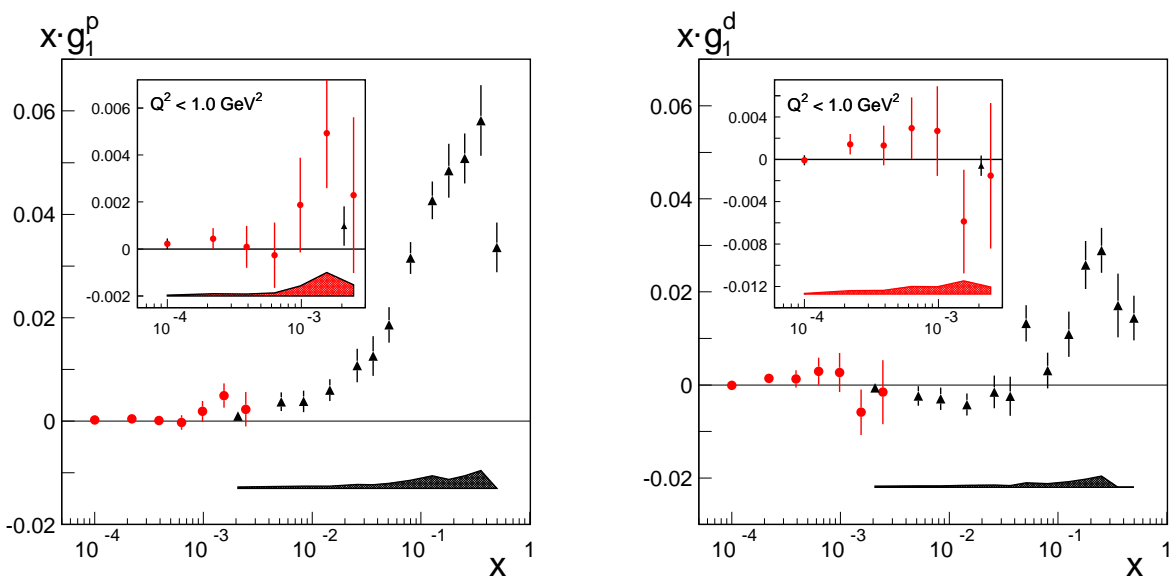

Figure 5: Structure functions, $x \cdot g_{1}(x)$, for the proton (left) and the deuteron (right) from the SMC [5]. The value of $Q^{2}$ is below $1 \mathrm{GeV}^{2}$ for $x<0.003$. 


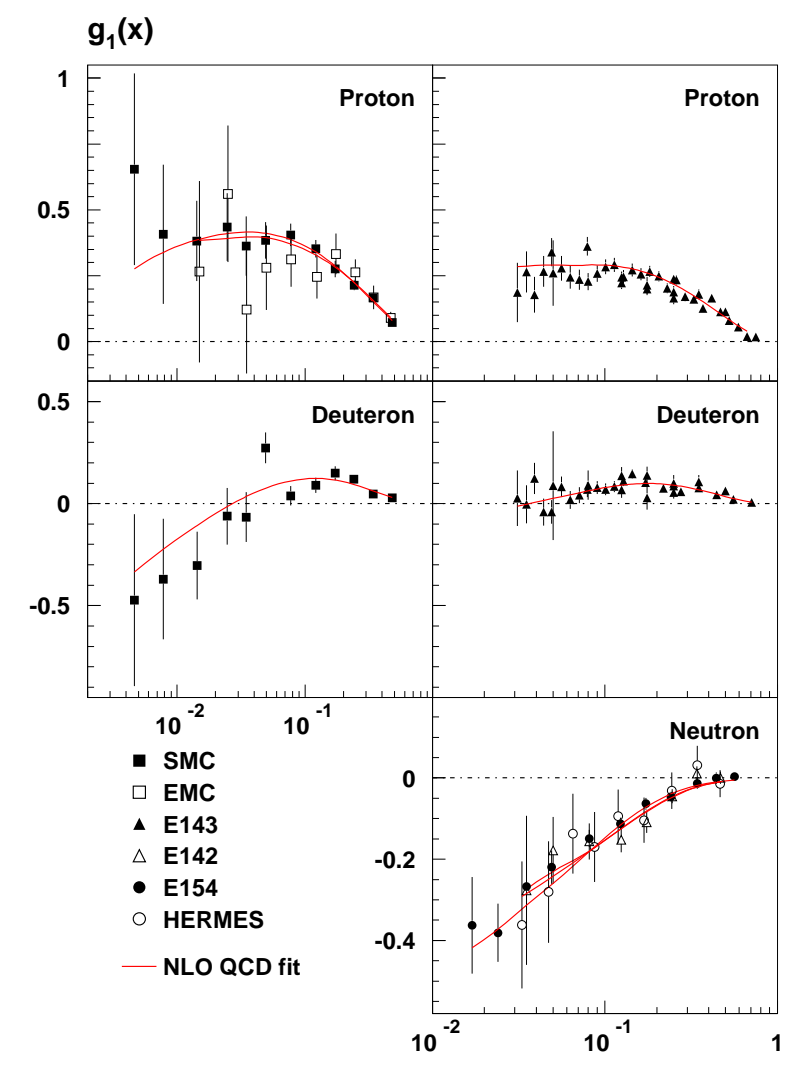

Figure 6: SMC next-to-leading order QCD fit to $g_{1}$ data of the proton, the deuteron and the neutron. The curves represent the fit for the different values of $Q^{2}$ of the data points 24.

While the non-singlet part of $\Gamma_{1}$ is scheme independent, the separation between gluon and quark singlet contributions is as usual ambiguous beyond leading order. The singlet part of $\Gamma_{1}$ is often denoted as $1 / 9 a_{0}\left(Q^{2}\right) C^{\mathrm{s}}\left(Q^{2}\right)$ with the "axial charge" $a_{0}$ (see Eq. 13). In the $\overline{\mathrm{MS}}$ scheme there is no explicit gluon contribution to $\Gamma_{1}\left(C^{\mathrm{g}}\left(Q^{2}\right) \equiv 0\right)$ and thus $\Delta \Sigma\left(Q^{2}\right)=a_{0}\left(Q^{2}\right)$ is scale dependent. The $\mathrm{AB}$ scheme is tailored to define a scale independent quark contribution, $\Delta \Sigma^{\mathrm{AB}}$, and to absorb the scale dependence in the anomalous gluon contribution to $a_{0}$

$$
a_{0}\left(Q^{2}\right)=\Delta \Sigma^{\mathrm{AB}}-n_{f} \frac{\alpha_{s}}{2 \pi} \Delta g\left(Q^{2}\right) .
$$

The gluon distribution function is the same in the two scheme discussed above. The quantity $\Delta \Sigma^{\mathrm{AB}}$ is often interpreted as the quark spin content of the nucleon. Therefore a large value of $\Delta \Sigma^{\mathrm{AB}}$ requires a large positive gluon polarisation in order to be 


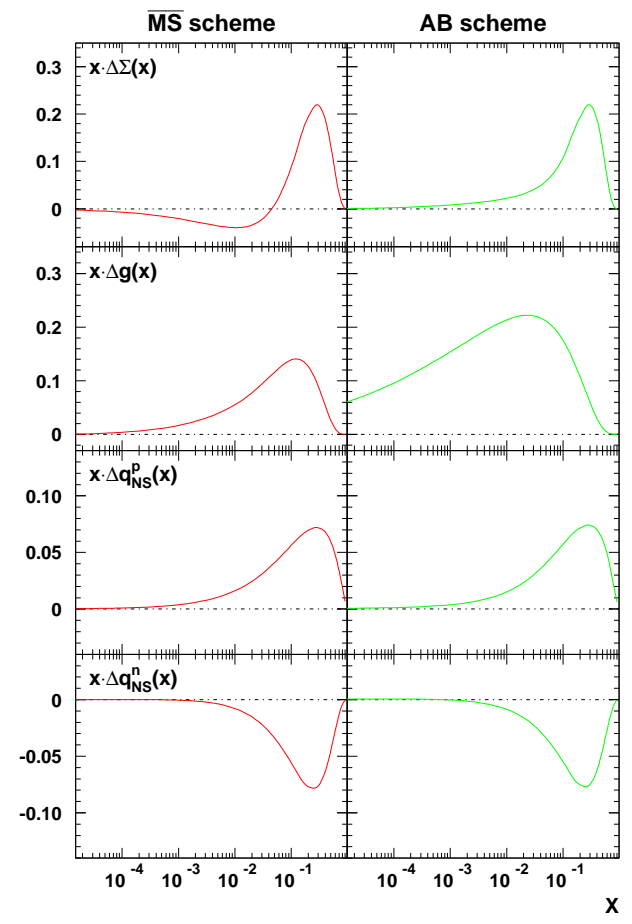

Figure 7: SMC next-to-leading order QCD fit, comparison of the fits in the $\overline{\mathrm{MS}}$ and $\mathrm{AB}$ schemes. The distributions are shown at $Q^{2}=$ $5 \mathrm{GeV}^{2}$ [24].
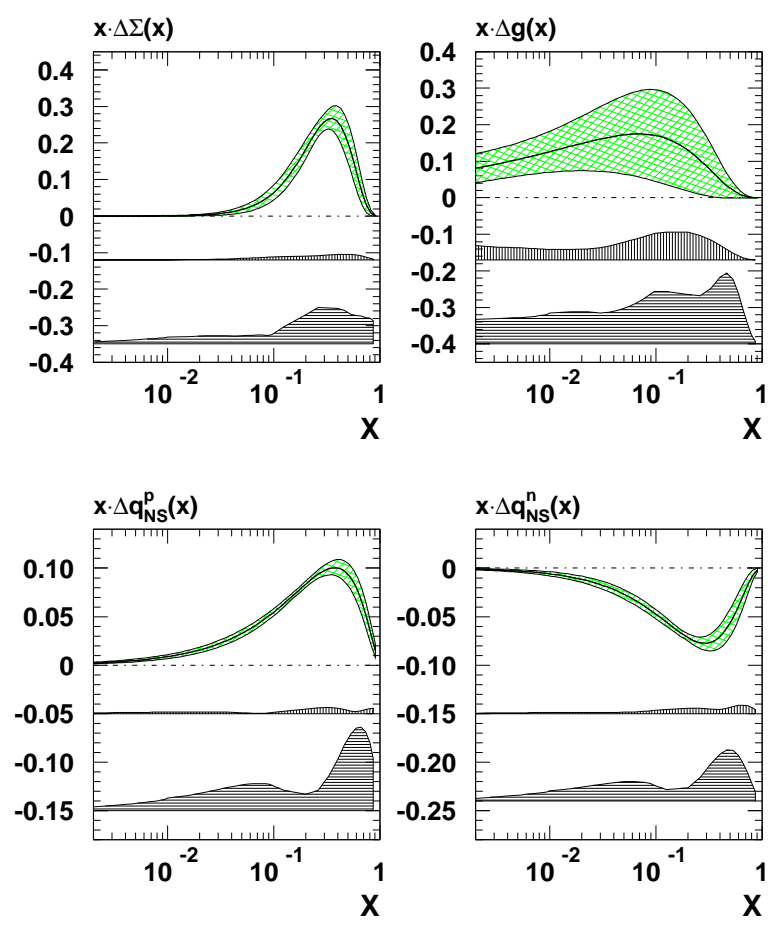

Figure 8: Uncertainties on the parton distribution functions. The cross-hatched band represents the statistical error only. The experimental (top) and theoretical (bottom) systematic errors are shown below the fitted distributions [24].

consistent with the small experimental value for $a_{0}$.

QCD analyses of the $g_{1}\left(x, Q^{2}\right)$ data were performed by several experimental [21, 24] and theoretical [25, 26, 27, 28, 29] groups. A particular difficult problem is the correct implementation of experimental and theoretical systematic errors. A detailed study of the propagation of these errors into the fitted parton distribution functions was carried out by the SMC [24]. Fits in $x$ and $n$ space, AB and $\overline{\mathrm{MS}}$ schemes, with different factorisation and renormalisation scales and different $Q_{i}^{2}$ were compared in this study. The $\overline{\mathrm{MS}}$ fit with $Q_{i}^{2}=1 \mathrm{GeV}^{2}$ to the proton, deuteron and neutron data is shown in Fig. 6. The parton distribution functions obtained in the $\overline{\mathrm{MS}}$ and $\mathrm{AB}$ schemes agree well for the non-singlet distributions (Fig. 团), while the $\Delta \Sigma(x)$ distributions must be different for a non-vanishing gluon distribution. The negative part of $\Delta \Sigma(x)$ in the $\overline{\mathrm{MS}}$ scheme leads a smaller first moment than the positive distribution in the AB scheme (see Eq. 12). The unexpected difference between the two gluon distributions reflects that the present data hardly constrain this quantity. For the other distribution functions the theoretical uncertainty dominates (Fig. (8). 
a) $\Delta \Sigma(\mathrm{AB})$
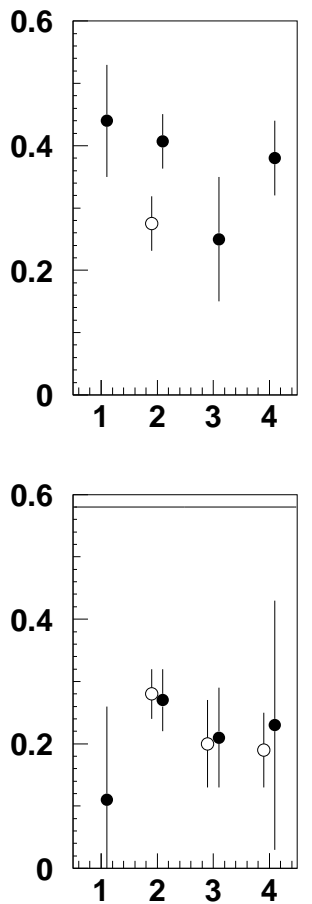

c) $a_{0}$ b) $\Delta g$
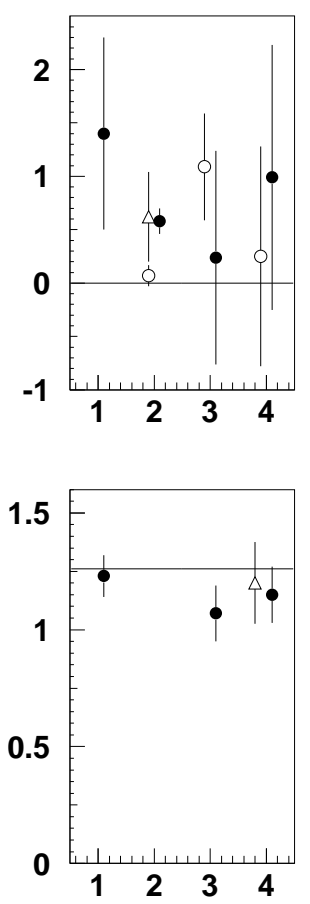

d) $\left|g_{a} / g_{v}\right|$

Figure 9: Moments at $Q^{2}=1 \mathrm{GeV}^{2}$ from QCD fits 1-4 [28, 29, 21, 24], a) "quark spin content", b) gluon polarisation, c) axial charge, Ellis-Jaffe sum rule, d) axial coupling, Bjorken sum rule.

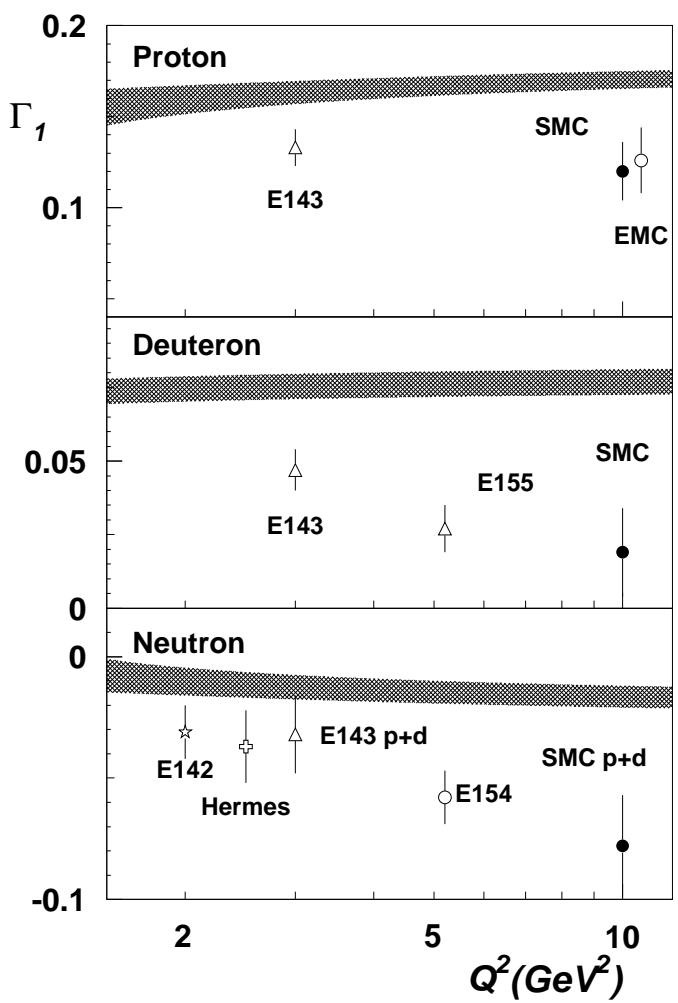

Figure 10: First moments of $g_{1}$ for the proton, the deuteron and the neutron at the typical $Q^{2}$ of the data. The hatched bands show the prediction of the EllisJaffe sum rule.

\section{Sum rules and moments}

From the QCD analyses the first moments and the axial charge can be determined directly by integration of the appropriate parton distribution functions. Figure 9 summarises the results from fits by (1) Altarelli, Ball, Forte, Ridolfi, Fit B [28], (2) Leader, Sidorov and Stamenov [29], (3) E154 [21] and (4) the SMC [24]. All fits use $Q_{i}^{2}=1 \mathrm{GeV}^{2}$, except the E154 fit which uses $Q_{i}^{2}=0.34 \mathrm{GeV}^{2}$.

The results for $\Delta g\left(1 \mathrm{GeV}^{2}\right)$ vary strongly, however all fits give positive values. Fit (2) only takes into account the statistical errors. A slight change of the parametrisation of the gluon distribution for this fit results in a quite different value for $\Delta g$ (open triangle, Fig. 9b). Often the violation of the Ellis-Jaffe sum rule is attributed to the axial anomaly and it is suggested that instead of the axial charge, $a_{0}$, the scale invariant quark spin content, $\Delta \Sigma^{\mathrm{AB}}$, should be consistent with the Ellis-Jaffe 
expectation of about 0.6. This is not supported by the data (Fig. 9a), which favour a value around 0.4 . In some fits the axial coupling constant $\left|g_{a} / g_{v}\right|$ was treated as a free parameter. The agreement with the experimental value of $1.2601 \pm 0.0025$ and thus with the Bjorken sum rule 30 is demonstrated in Fig. 9 $\mathrm{d}$. The moments obtained from the next-to-leading order fits take only correction to order $\mathcal{O}\left(\alpha_{s}^{2}\right)$ into account, while the corrections to the Ellis-Jaffe and Bjorken sum rules are known up to order $\mathcal{O}\left(\alpha_{s}^{3}\right)$ 31.

A direct evaluation of the first moment $\Gamma_{1}$ from the $g_{1}$ data requires the evolution of the data to a common value of $Q^{2}$ in the measured range and extrapolations to $x=0$ and $x=1$. While the latter is unproblematic, the small- $x$ extrapolation has long been debated in the literature. The presently most reliable procedure is to use the QCD fits for $x \rightarrow 0$. The present status of the world data for $\Gamma_{1}$ for the proton, the deuteron and the neutron is shown in Fig. 10. All experiments show a violation of the Ellis-Jaffe sum rule independent of the target. The three analyses using a QCD fit for the extrapolation to $x=0$ (SMC, E154, E155) show the strongest deviation due to the negative contribution from this extrapolation. This is most obvious for the neutron, where Regge-type extrapolations (E142, E143, Hermes) lead to first moments almost compatible with the Ellis-Jaffe value.

Assuming $\mathrm{SU}(3)$ flavour symmetry the first moment of $g_{1}$ is given by

$$
\Gamma_{1}\left(Q^{2}\right)=C^{\mathrm{s}}\left(Q^{2}\right) a_{0}\left(Q^{2}\right)+\frac{1}{12}\left(\left|\frac{g_{a}}{g_{v}}\right|-\frac{1}{3}(3 F-D)\right) C^{\mathrm{ns}}\left(Q^{2}\right) .
$$

In order $\mathcal{O}\left(\alpha_{s}^{3}\right)$ one obtains from the first moments the following values for the axial charge, $a_{0}: 0.12 \pm 0.15$ (SMC proton), $0.06 \pm 0.13$ (SMC deuteron), $0.18 \pm 0.10$ (E154 neutron) and $0.14 \pm 0.07$ (E155 deuteron). The precise E155 data dominate the average of $a_{0}=0.14 \pm 0.05$, which is somewhat lower than the value obtained directly from the QCD fits $a_{0} \simeq 0.20$ (Fig. 9 c). The agreement is satisfactory, in particular in view of the many differences in the procedures which include: the order in $\alpha_{s}$, SU(3) symmetry, fitting of $\left|g_{a} / g_{v}\right|$. For the evaluation of $a_{0}$ only those values of $\Gamma_{1}$ were considered, in whose evaluation a QCD extrapolation to $x=0$ was used.

\section{Status of $A_{2}$ measurements}

The structure function $g_{2}$ offers the unique possibility to study a pure twist-3 effect. It can be split into a twist-2 term, $g_{2}^{\mathrm{WW}}$ [32], which is calculable from $g_{1}$ and the twist-3 term $\bar{g}_{2}$

$$
\begin{aligned}
g_{2}\left(x, Q^{2}\right) & =g_{2}^{\mathrm{WW}}+\bar{g}_{2}\left(x, Q^{2}\right) \\
g_{2}^{\mathrm{WW}}\left(x, Q^{2}\right) & =-g_{1}\left(x, Q^{2}\right)+\int_{x}^{1} \frac{g_{1}\left(y, Q^{2}\right)}{y} \mathrm{~d} y .
\end{aligned}
$$




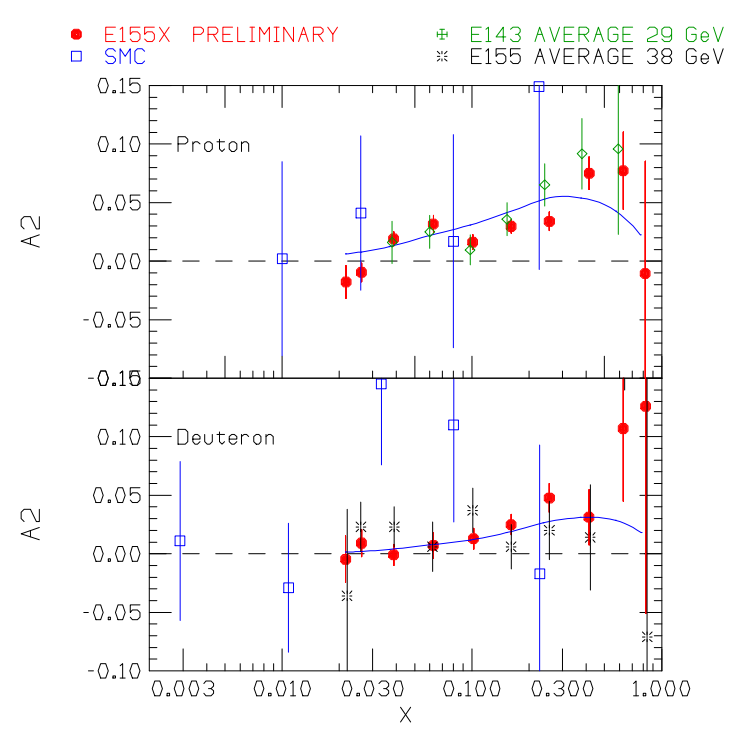

Figure 11: Preliminary $A_{2}$ asymmetries from the E155x experiment for the proton (top) and the deuteron (bottom) [34]. The solid line indicates the $A_{2}^{\mathrm{WW}}$ term.

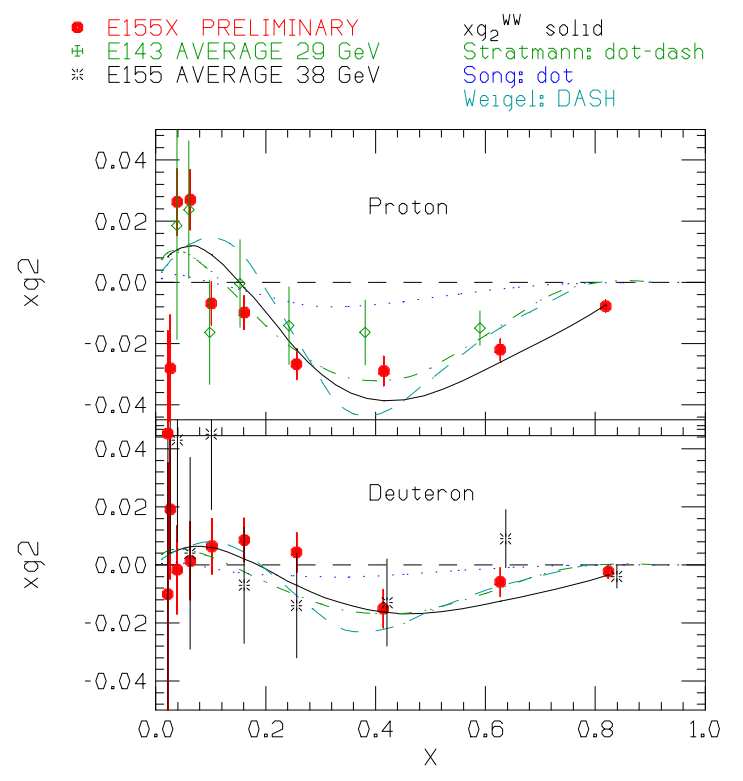

Figure 12: Preliminary E155x $x g_{2}(x)$ data for the proton (top) and the deuteron (bottom) [34]. Also shown are the $g_{2}^{\mathrm{WW}}$ term (solid) and several bag model calculations (dashed and dotted).

Measurements by the SMC [33] and by the E142 [7], E143 [8], E154 [10] and E155 [12] experiments showed that the corresponding asymmetry, $A_{2}$, is much smaller than its positivity limit $\left|A_{2}\right| \leq \sqrt{R}$. The very precise preliminary data from the dedicated $g_{2}$ experiment E155x [34, 20] now clearly establishes a non-vanishing $g_{2}$ for both, the proton and the deuteron. The data are in line with the Wandzura-Wilczek term, $g_{2}^{\mathrm{WW}}$, and any possible twist-3 term must be small (Figs. 11 and 12).

The twist-3 matrix element $d_{2}$

$$
d_{2}=3 \int_{0}^{1} x^{2} \bar{g}_{2}\left(x, Q^{2}\right) \mathrm{d} x
$$

is related to higher twist effects for the first moments and thus for the Bjorken sum rule. The world data are $d_{2}^{\mathrm{p}}=0.007 \pm 0.004$ and $d_{2}^{\mathrm{p}}=0.004 \pm 0.010$ for the proton and the neutron, respectively. All data come from the SLAC experiments E142-E155 and are shown in Fig. 13. The present precision is not sufficient to decide between bag model and QCD sum rule calculations. However, the precision expected for the E155x data might allow us to distinguish between the two classes of predictions. 


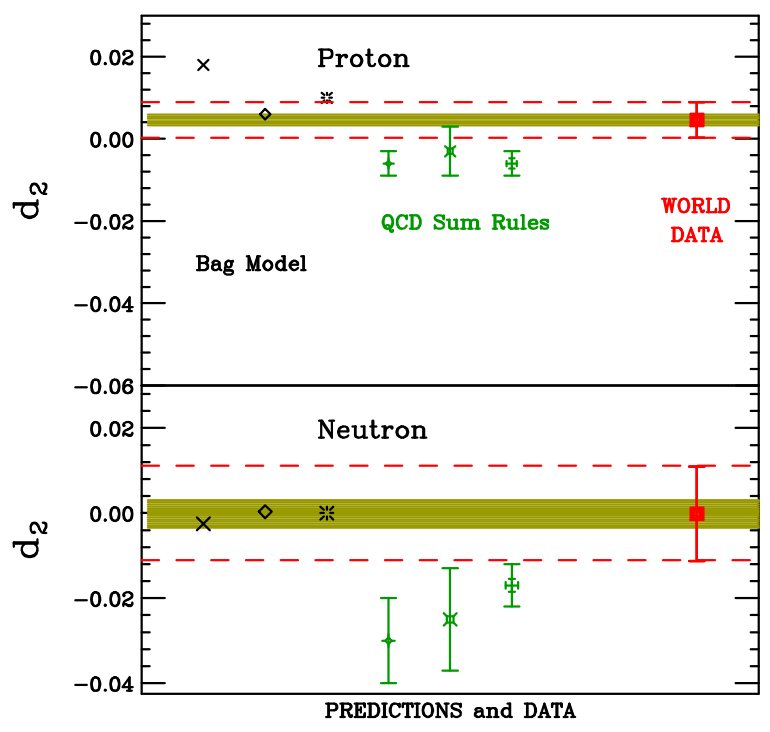

Figure 13: The twist-3 matrix element $d_{2}$ of the proton and the neutron. The dark band indicates the expected uncertainty of the E155x data, the values are not yet released. Also shown are bag model and QCD sum rule calculations [34].

\section{Semi-inclusive data}

In semi-inclusive processes a hadron is detected in addition to the scattered lepton. Due to the favoured fragmentation of e.g. a u-quark (d-quark) into $\pi^{+}\left(\pi^{-}\right)$one can perform a flavour separation in the parton distribution functions. The asymmetries from the SMC and Hermes experiments [6, 15] for positive and negative hadrons are shown in Fig. 14. A full particle identification was not available in these experiments. From these asymmetries one can derive the polarised valence parton distributions, $\Delta u_{\mathrm{v}}$ and $\Delta d_{\mathrm{v}}$, as well as the anti-quark distribution. For the latter in the Hermes analysis it was assumed that

$$
\frac{\Delta \bar{u}(x)}{\bar{u}(x)}=\frac{\Delta \bar{d}(x)}{\bar{d}(x)}=\frac{\Delta \bar{s}(x)}{\bar{s}(x)}
$$

The results are shown in Fig. 15 for the Hermes $x$ range, while the SMC extent to $x=3 \cdot 10^{-5}$. The Hermes systematic error is shown by the band, while that for the other data is included in the error bar.

Recently first results were reported involving azimuthal asymmetries. The SMC analysed data taken with transverse target polarisation in terms of the Collins angle and found an asymmetry of $A_{N}=0.11 \pm 0.06$ and $-0.02 \pm 0.06$ for positive and negative hadrons, respectively [35]. Hermes reported an azimuthal asymmetry of 
(a)

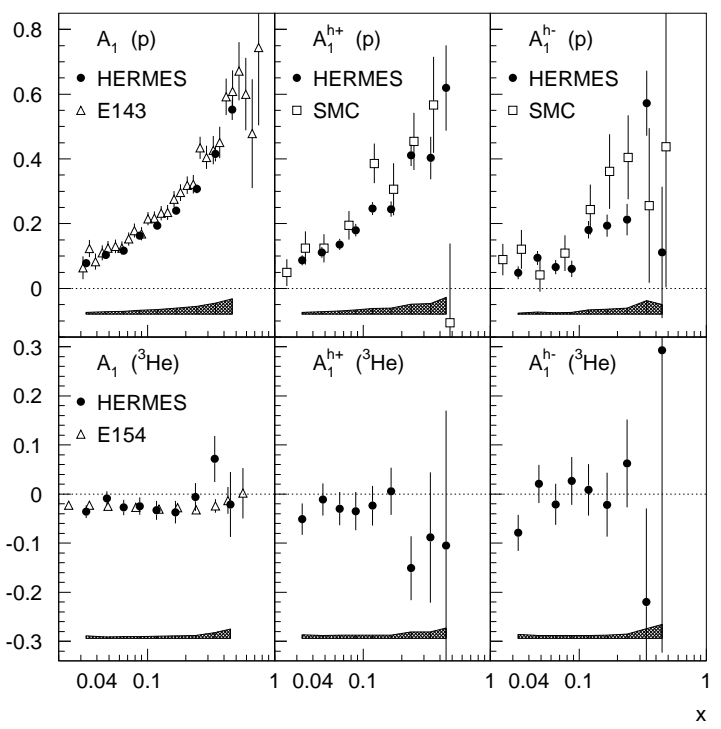

Figure 14: Inclusive (a) and semi-inclusive Asymmetries of positive (b) and negative (c) hadrons for the proton (top) and ${ }^{3} \mathrm{He}$ (bottom) [15].

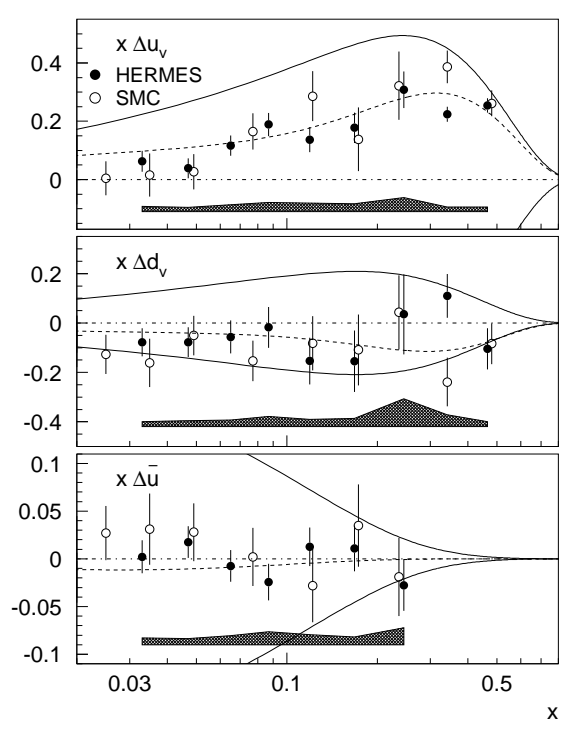

Figure 15: The valence and sea quark distributions at $Q^{2}=$ $2.5 \mathrm{GeV}^{2}$ from the $\mathrm{SMC}$ and Hermes [15]. Also shown are the positivity limit (solid) and a parametrisation from [25] (dashed).

$A_{N}=0.022 \pm 0.005 \pm 0.003$ for $\pi^{+}$and $-0.002 \pm 0.006 \pm 0.004$ for $\pi^{-}$using longitudinal target polarisation [36]. These data represent the first glimpse at the chiral-odd transversity structure function, $h_{1}$.

Hermes has studied asymmetries in events with high- $p_{T}$ oppositely-charged hadron pairs 37. The asymmetry is insignificant for events with positive hadrons with $p_{T}>1.5 \mathrm{GeV}^{2}$ and events where both hadrons have $p_{T}<1 \mathrm{GeV}^{2}$. For $p_{T}^{\mathrm{h}^{+}}>1 \mathrm{GeV}^{2}$ and $p_{T}^{\mathrm{h}^{-}}>1.5 \mathrm{GeV}^{2}$ there are two data points showing a non-zero asymmetry. The asymmetry in this region is $-0.28 \pm 0.12 \pm 0.02$. This has been interpreted in terms of a positive gluon polarisation.

\section{Summary and Outlook}

A large amount of high quality data is now available for the structure functions $g_{1}$ in a large range of $x$ and $Q^{2}$. The data are consistent with QCD evolution and the NLO fits taught us much about the polarised parton distribution functions. In contrast to the unpolarised case were neutrino scattering provides information on the flavour 
separation, in polarised deep inelastic scattering we must rely on the semi-inclusive processes using flavour tagging by hadrons. Semi-inclusive processes are also the only way in DIS to observe the chiral-odd structure function $h_{1}$, for which first information was presented this year. Big progress has been made in the knowledge of $g_{2}$.

In spite of all the precise data the spin puzzle is not yet resolved and a direct measurement of the gluon polarisation and if possible of the orbital angular momentum is inevitable. New experiments will study this quantity at CERN (COMPASS) and RHIC starting data taking 2001. An exciting longer-term perspective is the possibility of a polarised proton beam at Hera, which would open up a new window to the nucleon's spin structure.

I appreciate the help of E. Rondio and S. Rock and my colleagues from the SMC

and COMPASS collaborations in the preparation of this talk. The organizers of LP99 I thank for the invitation to this inspiring conference.

\section{References}

[1] J. Ashman et al. [European Muon Collaboration], Phys. Lett. B206, 364 (1988).

[2] J. Ashman et al. [European Muon Collaboration], Nucl. Phys. B328, 1 (1989).

[3] J. Ellis and R. L. Jaffe Phys. Rev. 9, 1444 (1974), Phys. Rev. 10, 1669 (1974).

[4] B. Adeva et al. [Spin Muon Collaboration], Phys. Rev. D58, 112001 (1998).

[5] B. Adeva et al. [Spin Muon Collaboration], Phys. Rev. D60, 072004 (1999).

[6] B. Adeva et al. [Spin Muon Collaboration], Phys. Lett. B420, 180 (1998).

[7] P. L. Anthony et al. [E142 Collaboration], Phys. Rev. D54, 6620 (1996).

[8] K. Abe et al. [E143 collaboration], Phys. Rev. D58, 112003 (1998).

[9] K. Abe et al. [E154 Collaboration], Phys. Rev. Lett. 79, 26 (1997).

[10] K. Abe et al. [E154 Collaboration], Phys. Lett. B404, 377 (1997).

[11] P. L. Anthony et al. [E155 Collaboration], Phys. Lett. B463, 339 (1999).

[12] G.S. Mitchell [E155 Collaboration], hep-ex/9903055.

[13] K. Ackerstaff et al. [HERMES Collaboration], Phys. Lett. B404, 383 (1997).

[14] A. Airapetian et al. [HERMES Collaboration], Phys. Lett. B442, 484 (1998). 
[15] K. Ackerstaff et al. [HERMES Collaboration], Phys. Lett. B464, 123 (1999).

[16] X. Ji, J. Tang, and P. Hoodbhoy, Phys. Rev. Lett. 76, 740 (1996).

[17] D. Adams et al. [Spin Muon Collaboration], Nucl. Instrum. Meth. A437, 23 (1999).

[18] T.D. Averett et al., Nucl. Instrum. Meth. A427, 440 (1999).

[19] K. Ackerstaff et al. [HERMES Collaboration], Nucl. Instrum. Meth. A417, 230 (1998).

[20] private communication, E155 homepage, http://www.slac.stanford.edu/exp/e155/home.htm].

[21] K. Abe et al. [E154 Collaboration], Phys. Lett. B405, 180 (1997).

[22] R. Mertig and W. L. van Neerven, Z. Phys. C70, 637 (1996).

[23] W. Vogelsang, Phys. Rev. D54, 2023 (1996).

[24] B. Adeva et al. [Spin Muon Collaboration], Phys. Rev. D58, 112002 (1998).

[25] T. Gehrmann and W. J. Stirling, Phys. Rev. D53, 6100 (1996).

[26] M. Gluck, E. Reya and W. Vogelsang, Phys. Lett. B359, 201 (1995).

[27] M. Stratmann, hep-ph/9907465.

[28] G. Altarelli, R. D. Ball, S. Forte and G. Ridolfi, Acta Phys. Polon. B29, 1145 (1998).

[29] E. Leader, A. V. Sidorov and D. B. Stamenov, Phys. Lett. B462, 189 (1999).

[30] J. D. Bjorken Phys. Rev. 148, 1467 (1966), Phys. Rev. D1, 1376 (1970).

[31] S. A. Larin, T. van Ritbergen and J. A. Vermaseren, Phys. Lett. B404, 153 (1997).

[32] S. Wandzura and F. Wilczek Phys. Lett. B72, 195 (1977).

[33] D. Adams et al. [Spin Muon Collaboration (SMC)], Phys. Lett. B336, 125 (1994).

[34] S. Rock, private communication, (1999).

[35] A. Bravar, Nucl. Phys. B (Proc. Suppl.) 79 , 520 (1999).

[36] A. Airapetian et al. [HERMES Collaboration], hep-ex/9910062.

[37] A. Airapetian et al. [HERMES Collaboration], hep-ex/9907020. 